\title{
II pretesto. Insegnare a leggere letteratura per realizzare di essere come tutti
}

\section{The pretext. Teaching to Read Literature in order to Realize that you are like everyone else}

\author{
Livia Radici Tavernese \\ SUPSI, Dipartimento formazione e apprendimento
}

ఐ livia.radici@supsi.ch

Riassunto / II presente contributo si propone di presentare un'attività didattica volta allo sviluppo e all'allenamento delle competenze di lettura e comprensione del testo letterario per allievi di 13/14 anni secondo un approccio induttivo. In particolare, l'attività è stata strutturata in tre momenti (preparazione alla lettura/ascolto, fruizione del testo, ritorno sul testo) e la riflessione degli allievi è stata stimolata attraverso l'uso di immagini e l'attenzione a locuzioni opportunamente individuate. Il confronto tra le ipotesi e le osservazioni degli allievi è risultato determinante allo scopo di entrare in empatia con i sentimenti evocati dal testo. Grazie all'apporto interpretativo attivo che i giovani lettori hanno offerto, si è realizzato il principio di intersoggettività e cooperazione tra autore e lettore.

Parole chiave: comprensione del testo; letteratura; approccio induttivo.

Abstract / This paper aims to introduce a teaching activity designed to improve reading literacy skills in 13- to 14-year-old students, using an inductive approach. For this activity three different parts are established: preparation for reading/listening, text fruition, and return to the text. Students are then invited to reflect on the meaning intended by the author by specifically identifying images and passages of the text. Comparing the different hypotheses and observations emerged from the students' discussions has been crucial for them to fully understand the emotions recalled by the text. Thanks to the students' contribution the cooperative principle in reading has been successfully realized.

Keywords: reading literacy skills; literature; inductive approach.

\footnotetext{
(C) 2021 Livia Radici Tavernese. Questo è un articolo Open Access, sottoposto a un processo di revisione tra pari a dop-pio cieco, pubblicato dal Centro competenze didattica dell'italiano lingua di scolarizzazione e dal Servizio risorse didattiche e scientifiche, eventi e comunicazione del DFA-SUPSI in collaborazione con l'Alta scuola pedagogica dei Grigioni. L'articolo è distribuito sotto i termini della Licenza Creative Commons Attribuzione - Non commerciale - Non opere derivate 4.0 Internazionale (CCBY-NC-ND 4.0).
} 
Il testo è intessuto di spazi bianchi, di interstizi da riempire e chi lo ha emesso prevedeva che essi fossero riempiti e li ha lasciati bianchi per due motivi. Anzitutto perché il testo è un meccanismo pigro (o economico) che vive sul plusvalore del senso introdottovi dal destinatario [...]. E in secondo luogo perché, via via che passa dalla funzione didascalica a quella estetica, un testo vuole lasciare al lettore l'iniziativa interpretativa, anche se di solito desidera essere interpretato con un margine sufficiente di univocità: un testo vuole qualcuno che lo aiuti a funzionare.

(Eco, 1979, pp. 51-52)

\section{Premessa}

Non è esperienza inconsueta quella di incontrare un testo letterario la cui comprensione non risulta immediata; ancor meno rara è la mancata percezione di tutte le sfumature implicite nel testo stesso. In tutti i casi - assai più frequenti per il lettore progrediente che per quello avvezzo - ad essere in gioco è, in definitiva, non solo l'apprezzamento estetico e la comprensione semantica del brano, ma anche un aspetto forse ancor più imprescindibile. Non penetrare pienamente la lettera, la figura, l'essenza di un testo impedisce che si realizzi la funzione più magica e sorprendente che la letteratura ricopre: se non si intende l'intentio auctoris, il sentimento che lo ha mosso a scrivere, lo stato d'animo che desidera condividere, non ci sarà coinvolgimento né riconoscimento, e senza questa agnizione non si realizzerà la catarsi, il dono più alto e motivante che la letteratura può elargire (Carnero, 2020, pp. 35-40). Quando si riesce a far inverare questa intenzione, insomma, ci si rende conto che il testo non è che un pretesto, un'occasione, uno straordinario strumento attraverso cui possiamo entrare in connessione con il sentire di un altro, renderci conto di cosa ci accomuna, di quanto, nonostante il tempo e lo spazio, le emozioni percorrano binari paralleli per tutti, di non essere i primi a trovarci in una certa situazione, di non essere soli, che gli altri sono come noi, che noi siamo come tutti.

\section{L'esperienza didattica}

L'esempio di trasposizione didattica che verrà illustrato di seguito, pur inqua- 
drato all'interno di un percorso più ampio, ha avuto luogo in una classe di allievi dai 13 ai 14 anni in due ore di lezione.

Il principio da cui parte è quello di permettere all'allievo di porsi di fronte all'opera letteraria con il numero di elementi indispensabili per avvicinarsi all'intenzione dell'autore in modo autonomo. A condurre l'avvicinamento al testo è stato un approccio il più possibile induttivo: sebbene guidata, l'esperienza di lettura si propone di realizzare il principio di intersoggettività e di cooperazione tra autore e lettore (Gerrig, 1993; Grice, 1975). La scelta della tipologia testuale, decisamente stratificata quanto ai livelli di lettura e ricca di elementi impliciti, vuole illustrare come anche un lettore poco esperto possa confrontarsi autonomamente con il poeta, se messo nelle giuste condizioni. Nel testo poetico, infatti, non sono necessariamente rispettate le condizioni (coerenza, presenza di relazioni causali e temporali esplicite, organizzazione gerarchica dei contenuti) che agevolano la rappresentazione semantica (Levorato, 2000).

Di seguito, alcuni passi fondamentali nell'avvicinamento del docente al testo, grazie ai quali la strutturazione della lezione si è rivelata efficace e gli allievi hanno potuto interagire con le parole dell'autore in maniera il più possibile autonoma. Necessariamente, per permettere agli allievi un'esperienza testuale autonoma, il docente si è dovuto concentrare sull'analisi dei processi di apprendimento che gli allievi sarebbero stati chiamati a mobilitare in ogni fase dell'attività1.

\section{Interroghiamo il testo, analizziamo il compito, prevediamo i processi: il do- cente studia come scomparire ${ }^{2}$}

Quella dell'analisi del testo e della preparazione della lezione è la fase di lavoro in cui il ruolo docente risulta più attivo ${ }^{3}$. La lettura del brano scelto attraverso

1 Un aspetto basilare in questa fase di lavoro è l'analisi delle caratteristiche del gruppo classe, delle sue abitudini di apprendimento e delle specificità di singoli allievi.

2 Nel corso del contributo, si farà ricorso più volte a questa iperbole con riferimento a un ruolo decentrato del docente, che ha assunto le vesti di consulente, tutor, coordinatore del lavoro svolto dagli allievi.

3 La pianificazione dell'intervento didattico prende le mosse a partire da determinati criteri, con variabili determinate dalla sensibilità di ciascun docente. Un approfondimento sulle operazioni che generalmente il docente è chiamato a fare è efficacemente proposto da Cisotto (2006, pp. 290-292). 
la lente delle competenze professionali ci mette, infatti, nella posizione di programmare l'intervento didattico secondo una prospettiva mimetica. Una volta individuati e selezionati gli elementi che risultano nodali per la comprensione del testo da parte degli allievi, ci si è chiesti quali fossero le strategie migliori per permettere agli allievi di scoprirli interrogando il testo in modo autonomo. Infatti, se reiterato, il percorso che porta il lettore ad attivare i processi necessari all'interazione con l'opera stimola il piacere della scoperta autonoma e personale, sostiene il senso di autoefficacia ${ }^{4}$ e, nei casi più virtuosi, può diventare soddisfazione, abitudine, ricerca della lettura.

L'esperienza testuale di cui si renderà conto ha come fulcro la lirica Lavandare di Giovanni Pascoli. Le considerazioni che da questa esperienza sono scaturite, però, provengono anche da precedenti trasposizioni didattiche e si sono rivelate utili pure in successive occasioni, durante la progettazione di interventi diversi da quello illustrato.

\subsection{L'importanza della parafrasi per il docente}

Quest'operazione ha agevolato notevolmente sia l'analisi del compito che avrebbero dovuto affrontare gli allievi sia la strutturazione dell'attività didattica, soprattutto grazie all'individuazione dei punti in cui lo scarto tra l'espressione poetica e il significato letterale risulta più evidente.

4 L'importanza della percezione, da parte del lettore, della propria competenza è un elemento fondamentale per la motivazione alla lettura e all'apprendimento (Cisotto, 2006, pp. 211-212). 


\section{Lavandare}

G. Pascoli

Nel campo mezzo grigio e mezzo nero resta un aratro senza buoi, che pare dimenticato, tra il vapor leggero.

E cadenzato dalla gora viene lo sciabordare delle lavandare con tonfi spessi e lunghe cantilene:

Il vento soffia e nevica la frasca, e tu non torni ancora al tuo paese! quando partisti, come son rimasta! come l'aratro in mezzo alla maggese. 10 5
Nel campo che è per metà arato e per metà no c'è un aratro senza buoi che sembra dimenticato, in mezzo alla nebbia.

E scandito dal canale che porta l'acqua al mulino si sente il rumore delle lavandaie che lavano i panni, sbattendoli, e le loro lunghe cantilene:

Il vento soffia e dai rami cadono le foglie, e tu non sei ancora tornato! da quando sei partito sono rimasta come un aratro abbandonato in mezzo al campo.

Grazie a questa operazione, emergono i punti del testo sui quali concentrarsi nella fase di strutturazione della lezione e ci si interroga su come, ove possibile, prevenire le perplessità degli allievi in fase di preparazione all'ascolto/lettura del testo, oltre che su come prevedere il ritorno sul testo dopo la lettura, per permettere il passaggio ai livelli di comprensione successivi.

In particolare, nel caso di specie, già al primo verso è rappresentato un elemento, il campo mezzo grigio e mezzo nero, la cui interpretazione non dev'essere immediata neanche per i lettori avvezzi alla realtà agricola, dunque lo è ancor meno per un allievo di scuola media; il campo è mezzo grigio e mezzo nero perché il suo terreno è stato dissodato solo per metà (la metà nera), e questo passaggio è indispensabile per permettere anche soltanto la comprensione del livello letterale. Inoltre, poiché questo elemento è l'immagine plastica dell'animo della protagonista della lirica (come sarà evidente all'ultimo verso), la sua comprensione risulta fondamentale perché il lettore sviluppi empatia con il sentimento descritto.

Su questo scenario si affacciano, nella seconda terzina, le lavandare, comparse sonore e mobili; pure con queste figure il giovane lettore non ha ormai più 
familiarità, e ancor meno può comprendere il loro manifestarsi ritmato dalla gora e prepotentemente sonoro, visto che i tratti che di loro sono dati sono prevalentemente percezioni uditive (lo sciabordare, i tonfi, le cantilene).

Ancora depistante può risultare il verso 7: la sola presenza del verbo nevicare, spesso frainteso dal giovane lettore nella sua accezione letterale anche a causa del magnifico uso in forma personale (nevica la frasca), in assetto chiastico rispetto al vento che soffia, tende facilmente a spostare l'immaginario verso la stagione invernale.

3.2 L'individuazione dei "trucchi" usati dall'autore per esprimersi efficacemente In questa fase, che coincide con l'analisi del testo, è stato necessario operare una selezione degli elementi indispensabili per gli allievi, affinché entrassero in empatia con l'autore. Gli elementi qui indicati, infatti, non esauriscono in alcun modo tutto quanto presente nel testo, ma sono risultati particolarmente degni di nota secondo la sensibilità del docente ${ }^{5}$.

Nel caso illustrato in questo contributo, ci si è concentrati su alcuni elementi relativi al livello semantico (in particolare, il campo semantico e le rime semantiche), a quello fonologico (onomatopee e allitterazioni) e a quello retorico-stilistico (similitudine e sinestesia).

\subsection{La previsione delle difficoltà di comprensione della lettera del testo}

Questo passaggio è necessariamente connesso con la parafrasi che è stata operata dal docente nella fase di avvicinamento al testo. In particolare, gli elementi su cui ci si è concentrati sono stati soprattutto quelli per cui è marcatamente evidente lo scarto tra il livello figurato e quello letterale, per l'interpretazione dei quali l'esplicitazione dell'implicito è indispensabile.

Un supporto affinché l'allievo possa confrontarsi autonomamente con il testo comprendendone almeno a grandi linee il messaggio complessivo deve, dun-

5 Non si ritiene, in questa sede, di soffermarsi su un'analisi metrica, fonologica, retorico-stilistica della lirica, già oggetto di autorevoli commenti e che chi legge è indubbiamente in grado di operare in autonomia. 
que, essere predisposto dal docente, possibilmente attingendo a strumenti suggestivi ma non risolutivi ${ }^{6}$.

L’alternativa per garantire la comprensione del testo e permettere di introdurre qualche elemento di analisi, naturalmente in un secondo momento, sarebbe quella di fornire una parafrasi già sviluppata, o di richiederla dotando il testo di note esplicative dei passaggi meno perspicui. Questa soluzione, ampiamente sperimentata nell'approccio tradizionale alla comprensione del testo, ha però tra i suoi deterrenti quello di limitare la centralità dell'allievo nell'approccio al testo, rendendo determinante la mediazione del docente.

\section{Conosciamo il testo, individuiamo il compito, realizziamo i processi: il do- cente struttura i materiali e riesce a scomparire}

A questo punto, dopo aver interrogato il testo e aver evinto, da questo esame, non solo le sue potenzialità, ma anche quali suoi snodi potrebbero mettere in difficoltà i giovani lettori, ci si è interrogati su come agevolare la comprensione del messaggio generale, con l'obiettivo di permettere agli allievi di arrivare alla prima lettura del testo con gli strumenti necessari per sciogliere le più importanti difficoltà di comprensione?

La fase di attività che è stata sfruttata maggiormente, in questo senso, è quella di preparazione all'ascolto. Si è scelto, per questo momento della lezione, di selezionare alcuni elementi particolarmente significativi (che nel caso del testo in oggetto coincidono con i luoghi che potrebbero comportare maggiore difficoltà di comprensione) e di anticiparli. Con questo stratagemma, si è potuto

6 L'adozione di un approccio strategico alla comprensione del testo è riconosciuto da più parti come la base per la progressione della competenza di lettura (De Beni \& Pazzaglia, 2001; De Beni et al., 2001). L’attività qui illustrata, posta all'interno di un percorso più articolato e programmato secondo lo stesso approccio, ha avuto, tra le altre finalità, quella di mettere gli allievi in condizione di monitorare la propria comprensione in maniera autonoma.

7 Il recupero delle conoscenze del lettore durante la lettura concorre inevitabilmente alla costruzione della rappresentazione del testo e del suo messaggio (Van den Broek \& Gustafson, 1999). Van Dijk e Kintsch (1983) sostengono l'importanza di questo processo, introducendo il concetto di modello mentale della situazione, in base al quale una rappresentazione ottenuta attraverso l'attivazione di elementi già noti è più povera di dettagli, ma più ricca di quelle conoscenze che il testo lascia solo presupporre e il lettore recupera dalla sua personale enciclopedia del mondo. 
ottenere un duplice effetto positivo: quello di incuriosire gli allievi sulla natura e sul messaggio del testo che si apprestavano a leggere e quello, fondamentale ai fini della comprensione della lettera del testo e dei suoi livelli ulteriori, di comprendere, evocare e visualizzare con immediatezza lo scenario descritto. A questo effetto ha indubbiamente contribuito la scelta del testo che, secondo l'uso pascoliano, presenta oggetti o paesaggi speculari rispetto allo stato d'animo descritto.

\subsection{L'ideazione delle strategie di agevolazione alla comprensione del testo} Per immaginare la fase di preparazione all'ascolto, si può ipotizzare l'uso di diversi codici alternativi, tra i quali:

- quello testuale (anticipazione di pericopi di testo, opportunamente scelte);

- quello uditivo (diffusione di suoni evocativi e pertinenti con l'ambientazione di riferimento);

- quello iconico (esposizione di immagini rappresentative di uno o più elementi citati nel testo).

La lirica di Pascoli, come spesso accade per altri testi dello stesso autore, rappresenta situazioni e oggetti la cui visualizzazione è indispensabile per la comprensione del messaggio complessivo e dell'intenzione dell'autore. Inoltre, alla luce di quanto individuato in fase di parafrasi, i punti potenzialmente oscuri del testo sono risultati facilmente rappresentabili attraverso fotografie e immagini; in particolare nel caso dell'aratro citato nel testo, le immagini sono risultate un eccezionale facilitatore per la comprensione dei significati letterali e figurati del testo, oltre che dello stato d'animo che l'autore desiderava rappresentare. Per questi motivi, per la fase di preparazione all'ascolto, si è prediletto l'uso di immagini. Nella fase di ritorno sul testo, invece, la ripresa di alcune formule ha permesso di focalizzare l'attenzione su luoghi testuali precisi.

\subsection{La preparazione dei materiali}

Questa fase del lavoro preparatorio è la sintesi e la fusione di tutti gli elementi e le suggestioni raccolti dal docente durante il suo percorso di avvicinamento 
al testo e si concretizza nella realizzazione delle schede destinate agli allievi (Allegato 1). Si tratta di prestare attenzione alla scelta delle immagini (fase di preparazione all'ascolto/lettura) e alla selezione dei luoghi testuali sui quali concentrarsi (fase di ritorno sul testo).

Perché il docente e le sue indicazioni esplicite scompaiano, quindi per immaginare un approccio di tipo sostanzialmente induttivo, è necessario che le immagini scelte siano più d'una per ciascun elemento e rechino indizi progressivi. In particolare, è stata individuata questa sequenza:

- per l'aratro: l'oggetto decontestualizzato ma visibile in ogni sua parte (Figura 1); l'oggetto nel suo contesto ma non in uso (Figura 2); l'oggetto in uso in un campo la cui terra reca differenze cromatiche tra la parte lavorata e quella brulla (Figura 3);

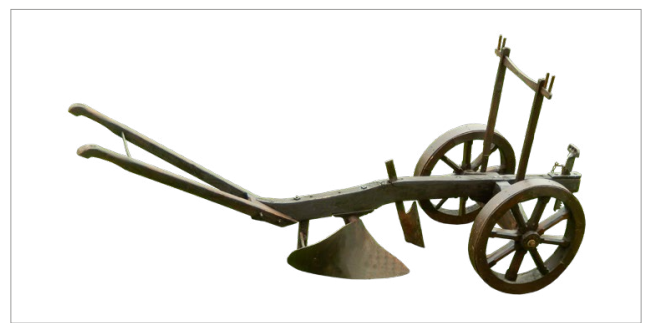

Figura 1. Un aratro decontestualizzato, ben visibile in ogni suo componente (fonte: pixabay.com).



Figura 2. Un aratro in disuso su un campo (fonte: pixabay.com). 


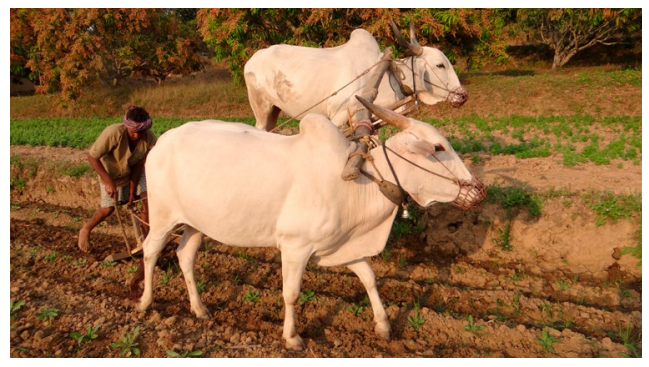

Figura 3. Un aratro in uso su un campo la cui terra reca differenze cromatiche tra la parte dissodata e quella brulla (fonte: pixabay.com).

- $\quad$ per le lavandaie: un lavatoio privo d'acqua, visibile in ogni sua parte (Figura 4); un lavatoio colmo d'acqua con le fontane in evidenza, ma non in uso (Figura 5); la struttura in uso da parte delle lavandaie, in posa in una foto d'epoca (Figura 6); le lavandaie riprese all'opera al bordo di un canale, possibilmente intente in chiacchiere o canti $^{8}$ (Figura 7).

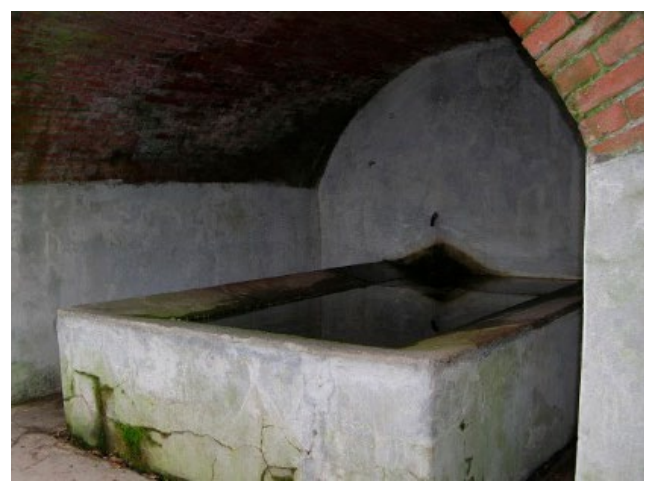

Figura 4. Un lavatoio privo d'acqua, visibile in ogni sua parte (fonte: Bbruno, CC BY-SA 3.0, via Wikimedia Commons).

8 Si è inteso inserire le immagini relative al lavatoio, pur assente nel testo, per due motivi: permettere agli allievi di rileggere, eventualmente, il territorio dove sono talora ancora presenti strutture analoghe e facilitare la realizzazione dell'immagine di più donne riunite in un unico compito. 


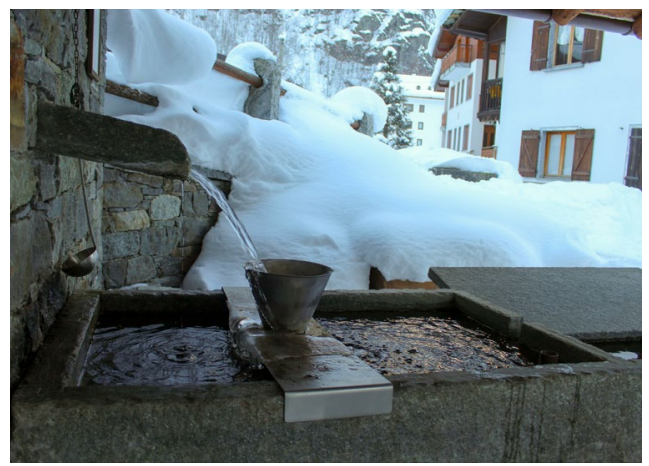

Figura 5. Un lavatoio colmo d'acqua con le fontane in evidenza, non in uso.

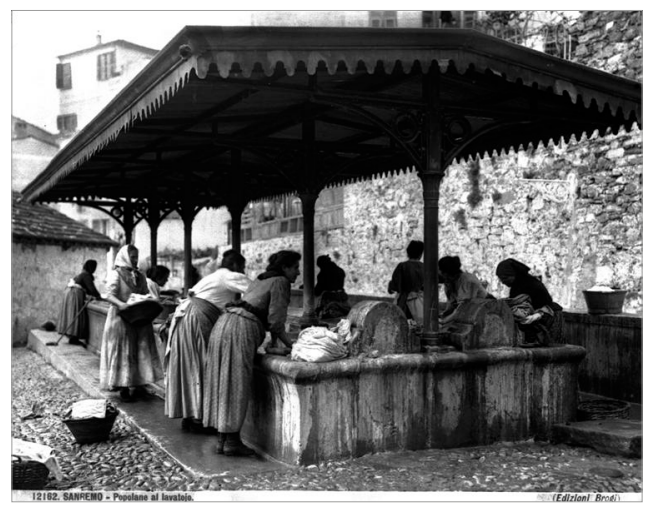

Figura 6. La struttura in uso da parte delle lavandaie, in posa in una foto d'epoca (fonte: Wikipedia).



Figura 7. Le lavandaie riprese all'opera al bordo di un canale, intente in chiacchiere o canti (fonte: Wikipedia). 
Questa successione di immagini, proposte una per volta, ha stimolato gli allievi ad osservare con attenzione, ad attivare la produzione di inferenze, ad intuire, a formulare ipotesi che, nel confronto tra l'immagine precedente e quella sottoposta in un secondo momento, sono state riviste, corrette, inquadrate nuovamente alla luce di ulteriori indizi. Perché questo avvenisse, l'esposizione delle immagini è stata immediatamente seguita da domande-guida, non suggestive, strutturate con l'intenzione di attingere al bacino delle conoscenze già in possesso dei giovani lettori e di restringere progressivamente il campo.

Naturalmente, accanto al meccanismo virtuoso di scoperta dei singoli elementi oggetto di attenzione, corrono parallele la crescente curiosità in merito al testo che ci si accinge ad affrontare e la raccolta, inconscia ma efficace, di conoscenze necessarie alla comprensione del messaggio.

La posizione e la struttura degli spazi di compilazione da parte degli allievi merita una riflessione a parte; nell'esperienza di chi scrive, infatti, questa soluzione si è rivelata efficace in diversi frangenti. Visto che spesso, posti di fronte alle righe preposte dal docente per permettere di rispondere a singoli quesiti, gli allievi si lasciavano impressionare e condizionare dal numero e dalla spaziatura delle linee, deducendone magari una determinata aspettativa da parte del docente, si è voluto sperimentare, e con successo, questo formato che lascia ciascuno libero di interpretare a suo modo il livello di approfondimento della risposta e ha l'ulteriore pregio di stimolare a compilare riempiendo lo spazio con precisazioni ulteriori.

\subsection{II ritorno sul testo}

Una volta affrontata la lettura (o l'ascolto) del testo, che è risultata di più facile accesso grazie alla fase di preparazione, ci si è concentrati su elementi selezionati a discrezione del docente, allo scopo, da un lato, di valorizzare alcuni aspetti testuali ritenuti particolarmente significativi e, dall'altro, di integrare correttamente la programmazione prevista per la classe mantenendo un approccio di tipo induttivo. Ne siano esempio le domande-guida elaborate con il fine di lasciare l'allievo libero di articolare il proprio pensiero, ma anche di articolare una riflessione su particolari impliciti del testo (Dov'è ambientata la scena? Quali elementi sono presenti sulla scena? In che stagione siamo e da cosa 
lo possiamo intuire? Chi parla? A chi parla?). I quesiti posti, in effetti, si sono dimostrati un efficace strumento di differenziazione: le risposte attese potevano variare dalla manifestazione della comprensione letterale del testo a riflessioni ben più articolate. In ogni caso, grazie a queste domande, tutti gli allievi si sono potuti concentrare su dettagli trascurati a una prima lettura, ma fondamentali per meglio comprendere e semmai confermare l'interpretazione del messaggio.

Allo stesso modo, dopo una seconda lettura autonoma del testo gli allievi sono stati stimolati a concentrare la propria attenzione sul livello retorico e su quello metrico attraverso un approccio induttivo. Alcune di queste consegne, che richiedevano una lettura più capillare del testo e stimolavano la ricerca, il riconoscimento e l'analisi di singole locuzioni, sono state svolte da ciascun allievo in maniera autonoma (Quali parole ti suggeriscono dei suoni? Sottolineale. Sottolinea con un altro colore le parole che producono una rima. Che schema ha usato il poeta? Secondo te, tra le parole che fanno rima c'è un rapporto di significato?). Invece, è stato previsto un momento di confronto con un compagno nei casi in cui il quesito era maggiormente aperto, non mirato all'identificazione diretta di un determinato aspetto del testo ma formulato con un intento maggiormente evocativo (Rileggi questo verso: "lo sciabordare delle lavandare". Cosa puoi notare? Analizza quest'espressione: "tonfi spessi”. Noti qualcosa di particolare?).

Infine, dopo un momento di lettura conclusivo, durante il quale ciascuno ha potuto apprezzare il componimento alla luce di quanto notato durante le precedenti fasi della lezione, gli allievi si sono confrontati nuovamente, in coppia o in piccolo gruppo 9 , per considerare i luoghi che, giunti a quel livello di conoscenza del testo, hanno trovato più suggestivi rispetto alle emozioni che l'autore voleva veicolare. In un ultimo momento di fruizione autonoma, poi, ciascun allievo ha cercato di sintetizzare il messaggio evinto dal testo, concentrandosi sull'aratro, simbolo di uno stato d'animo netto e centrale nella lirica. Non è un

9 La motivazione alla comprensione e all'identificazione con il testo è potenziata dal lavoro collaborativo, che contribuisce notevolmente anche alla costruzione di strategie personali e favorisce il processo di acquisizione della conoscenza attraverso l'ampliamento di visuali, relazioni e punti di vista sui saperi (Cisotto, 2006, p. 228). 
caso, d'altronde, se Pascoli lo ha voluto, in una composizione ad anello, tanto nel primo quanto nell'ultimo verso.

Al termine di ciascuna di queste fasi di lavoro, la classe ha condiviso le proprie osservazioni e il docente ha assunto il compito di ascoltare, accogliere attraverso l'uso della lavagna e rilanciare i contributi degli allievi. In diversi casi, quando le osservazioni erano particolarmente sentite o acute e quando gli elementi evidenziati facevano riferimento al livello retorico del testo, il docente ha fornito spiegazioni ulteriori o ha segnalato agli allievi la scoperta di un particolare tropo e la funzione per cui, generalmente, questo viene usato, contribuendo alla contestualizzazione dell'elemento nel testo.

\section{Conclusioni}

Una delle motivazioni che ha portato alla scelta di questo testo, oltre all'indiscusso valore letterario che lo permea e alla ricchezza di stimoli che porta con sé, era una convinzione precisa: purtroppo, non uno di noi nell'arco di un'esistenza (e questo è ancor più vero se si pensa ai patimenti dell'adolescenza) può dirsi alieno dal senso di inutilità e di abbandono descritto da Pascoli. A tutti è capitato, nostro malgrado, di sentirci svuotati, senza scopo, senza risorse, padroni di nulla e in nulla padroni di noi, proprio come quell'aratro, in un campo che non si può seminare perché arato solo per metà, senza buoi che lo trainino, senza possibilità di muoversi da solo, senza nessuno che abbia bisogno di lui.

Nelle settimane successive alla lettura di questo testo, stimolati a esercitare l'inventio a partire da uno stimolo, gli allievi hanno espresso con una similitudine, una metafora o un'analogia una situazione in cui si erano sentiti (o si sarebbero potuti sentire) abbandonati e senza risorse. Diversi allievi hanno trovato immagini straordinariamente eloquenti, grazie alle quali si può intravedere l'identificazione con le sensazioni evocate in loro dal testo della lirica pascoliana: «un'auto nel deserto senza benzina», «una lama consumata che non taglia più», «una biro scarica che non ha inchiostro quando devo scrivere», «una matita spuntata quando non hai il temperino», «un ombrello distrutto dal vento forte nella tempesta e non c'è un tetto per ripararti».

La speranza è che si trovi sempre più spesso una chiave per mostrare ai nostri giovani lettori che la letteratura è la risorsa suprema per non sentirci mai soli: una raccolta di sensazioni già provate da un altro che era come noi. 


\section{Bibliografia}

Carnero, R. (2020). Il bel viaggio. Insegnare letteratura alla generazione Z. Milano: Bompiani.

Cisotto, L. (2006). Didattica del testo. Processi e competenze. Roma: Carocci.

De Beni, R., Cisotto L., \& Carretti B. (2001). Psicologia della lettura e della scrittura. Trento: Erickson.

De Beni, R., \& Pazzaglia F. (2001). Lettura e metacognizione. Attività didattiche per la comprensione del testo. Trento: Erickson.

Eco, U. (1979). Lector in fabula. La cooperazione interpretativa nei testi narrativi. Milano: Bompiani.

Gerrig, R. J. (1993). Suspense in the Absence on Uncertainty. Journal of Memory and Language. 28, 663-48.

Grice, P. (1975). Logic and Conversation. In P. Cole \& J. L. Morgan (a cura di), Syntax and Semantics. 3 Speech Acts (pp. 41-58). New York: Academic Press.

Levorato, M. C. (2000). Le emozioni della lettura. Bologna: il Mulino.

Van den Broek, P., \& Gustafson, M. (1999). Comprehension and Memory for Texts; Three Generations of Reading Research. In S. R. Goldman, A. C. Graesser \& P. Van den Broek (a cura di), Narrative Comprehension Causality and Coherence. Essays in Honor of Tom Trabasso (pp. 15-34). Mahwah (NJ): Erlbaum.

Van Dijk T. A., \& Kintsch W. (1983). Strategies of Discourse Comprehension. New York: Academic Press. 